\title{
Assessing the accuracy and confidence of healthcare professionals in recognising that a patient is so unwell they might die during a hospital admission
}

\author{
Authors: Sarah Case, Philippa Dawson, James Morris and Miranda Cole
}

\section{Background}

Acting upon the early recognition of dying facilitates advanced care planning, allowing better end-of-life (EOL) care to be delivered in more appropriate settings. ${ }^{1}$ Commonly cited barriers to discussing EOL care with patients include fear of causing distress, lack of confidence and limited time in an increasingly stretched healthcare system. ${ }^{2}$ These barriers are evident locally: late transition to EOL care was a theme highlighted by a recent mortality review at Bristol Royal Infirmary, UK. Therefore, we aimed to:

$>$ evaluate healthcare professionals' accuracy in recognising a patient is so unwell they might die that admission

> collect information about staff perceptions of healthcare professionals' ability to recognise dying

$>$ develop a teaching intervention to address identified needs.

\section{Methods}

We asked nurses and doctors, 'Is this patient so unwell they might die on this admission?' on an acute medical ward, geriatric ward and oncology ward, and correlated their answers with the patient's survival to discharge. A subgroup of staff provided their opinion on how accurate and confident they felt they and their colleagues are in recognising dying, and subsequently small group teaching for nursing staff was held to increase knowledge and skills in caring for dying patients.

\section{Results and discussion}

Combining all available responses for all staff members and 149 patients, the overall sensitivity and specificity of our question was $56 \%$ and $95 \%$, respectively, with a positive predictive value of $68 \%$. Qualitative data suggested staff felt they and their colleagues were confident recognising dying, but there were barriers in acting upon this. At time of abstract submission results for the oncology ward were still pending.

\section{Conclusion}

Healthcare professionals were reasonably accurate in predicting whether patients would die during an admission. Despite confidence in this area there were barriers preventing staff acting upon their concerns. We feel our results could be used to empower healthcare professionals to act upon their instincts, a valuable step in improving the provision of EOL care to dying patients.

\section{Conflict of interest statement}

None declared.

\section{References}

1 Brinkman-Stoppelenburg A, Rietjens JA, van der Heide A. The effects of advanced care planning on end-of-life care: a systematic review. Palliat med 2014;28:1000-25.

2 Royal College of Physicians. Talking about dying: how to begin honest conversations about what lies ahead. London: RCP, 2018. 